\title{
On Anomalous Fluorescence of Symmetrical Polymethine Dyes
}

\author{
P. O. Kondratenko ${ }^{1}$ and Yu. M. Lopatkin $\mathbb{D}^{2}$ \\ ${ }^{1}$ Department of Theoretical and Applied Physics, Aerospace Institute, National Aviation University, \\ 1 Cosmonaut Komarov Avenue, Kyiv 03680, Ukraine \\ ${ }^{2}$ Department of Electronics, General and Applied Physics, Sumy State University, 2 Rimsky-Korsakov Street, \\ Sumy 40007, Ukraine
}

Correspondence should be addressed to Yu. M. Lopatkin; yu.lopatkin@gmail.com

Received 22 September 2019; Revised 3 March 2020; Accepted 10 April 2020; Published 14 May 2020

Academic Editor: Wonho Jhe

Copyright ( 92020 P. O. Kondratenko and Yu. M. Lopatkin. This is an open access article distributed under the Creative Commons Attribution License, which permits unrestricted use, distribution, and reproduction in any medium, provided the original work is properly cited.

\begin{abstract}
The group-theoretical analysis of polymethine dyes (PMD) showed that relaxation processes between the states $S_{3}$ and $S_{1}$ are forbidden, either by radiation or by internal conversion. From the state $S_{3}$, only transition to the ground state of the molecule is possible. Since the experimental data state that the quantum yield of $S_{3} \longrightarrow S_{0}$ fluorescence does not exceed $1 \%$, it is indicated that the internal conversion rate can be 2 orders of magnitude higher than the radiative relaxation rate of the molecule. Concerning the reasons for the appearance of fluorescence from the higher excited states of molecules, it can be asserted that the necessary condition for the appearance of $S_{3} \longrightarrow S_{0}$ fluorescence is the absence of $S_{0} \longrightarrow S_{1}(v)$-absorption in the region of the $S_{0} \longrightarrow S_{3}$ transition. The sufficient condition is the corresponding symmetry of the excited states, which imposes a prohibition on the $S_{3} \longrightarrow S_{1}$ relaxation process.
\end{abstract}

\section{Introduction}

For a long time, scientists believed that azulene is the only compound with anomalous fluorescence and band in the radiation spectrum above the first absorption band of the molecule [1]. The synthesis and studies of new dyes have shown that azulene is far from being unique in this regard [2].

The ketone radical of the azaxantone AXH. showed double fluorescence peaks at 460 and $645 \mathrm{~nm}$, which were assigned to the transitions $D_{2} \longrightarrow D_{0}$ and $D_{1} \longrightarrow D_{0}$, respectively [3]. It was found that the fluorescence lifetime for $D_{2} \longrightarrow D_{0}$ (1.0 ns) was longer than that for $D_{1} \longrightarrow D_{0}$ $(0.4 \mathrm{~ns})$. The fluorescence quantum yields $D_{1} \longrightarrow D_{0}$ and $D_{2} \longrightarrow D_{0}$ were estimated at $0.0008 \pm 0.0002$ and $0.05 \pm 0.02$, respectively. These abnormal emitting properties can be attributed to the pyridine ring in Azaxantone. AXH. is a new example of a neutral radical that violates Kasha's rules.

Two bands are observed in the radiation of 2-butylamino6-methyl-4-nitropyridine N-oxide (2B6M) [4]. Relative intensities of the bands depend on the wavelength of the excitation that violates Kasha-Vavilov's rule. The aim of the study was interpretation of these results. Based on the measured lifetimes and quantum fluorescence yields, a kinetic scheme was compiled to explain the experimental results.

Tryptophan was one of the first organic compounds for which a violation of Vavilov's law was also established [5]. This law is concerned by the independence of the quantum yield of fluorescence from the frequency of exciting light. Anomalous $S_{2} \longrightarrow S_{0}$ fluorescence of tryptophan in aqueous ethanol solutions was detected as a result of the transition from the second singlet-excited level to the ground level. The violation was explained by the competition of ultrafast (at least $10 \mathrm{fs}$ ) nonradiative deactivation and the photo-induced electron transfer processes occurring in highly excited singlet $S_{n}(n>1)$ states of the amino acid. Using indirect methods and primarily fluorescence, one can obtain a qualitative picture of the processes occurring in $S_{n}$ states.

Recent achievements in the synthesis of multicomponent nanocrystals have made it possible to create the highly luminescent molecular nanocrystal with unique photophysical behavior and functionality. This functionality is due to the weak vibronic coupling between the excited states, which thereby violates Kasha's rule by radiation from several excited levels [6]. Using single-particle photoluminescent spectroscopy, it was 
shown that the transition from the higher energy level through spatially indirect recombination is possible in addition to the expected radiation transition from LUMO to HOMO.

During these three or four decades, significant progress has been made in the accumulation of abnormal emission spectral data of organic molecules, including emission from higher excited states. In particular, experiments provided information on the internal photophysical properties of cooled and isolated molecules in which the Boltzmann distribution is practically negligible. Organic molecules that have emitting higher excited states form unique systems. Application of a number of modern methods to these systems should provide more detailed information about their photophysical properties. These data will bring a deeper understanding of the dynamic behavior of isolated molecules in their excited states. At least three mechanisms of the emergence of fluorescence from higher excited states have been proposed in the literature [7]: (A) fluorescence occurs through thermal reexcitation of a molecule from a lower excited state, for example, $S_{1}$; (B) this occurs through the inverse internal transformation from the lower singlet state in the absence of collisions; and (C) it originates directly from a higher singlet state without the participation of a fluorescent component through inverse internal conversion from a lower singlet state, i.e., fast fluorescence. In case (C), the rate of internal conversion from a higher singlet state to a lower state is considered to be slow enough to compete with fluorescence from a higher state.

The manifold of explanations for the nature of the occurrence of abnormal fluorescence corresponds to the manifold of its manifestations.

One of the classes of dyes in which a violation of Kasha's rule has been found is polymethine dyes [8-10]:<smiles></smiles>

A characteristic feature of polymethine dyes (PMD) is the presence of an odd number of methine groups $(\mathrm{CH}=)$. At the ends of the polymethine chain, there are fragments (identical or different) that determine the properties of the dye. Depending on the nature of the substituents $R$, one can distinguish cyanine (both substituents are the same), hemicianine (one substituent has electron-donor properties, and the second has electronacceptor properties), carbocyanine dyes, and so on.

As a rule, in the absorption spectrum of PMD, there is a long-wavelength intense band, the position of which substantially depends on the amount of methine groups in the polymethine chain. There is a sufficiently large gap between the first absorption band and the subsequent ones, where absorption is practically absent. Then, weak bands and the intense absorption ones in the UV region appear. It turned out that some molecules from a large class of PMD luminesce in the transparency region of the dye between the first and subsequent absorption bands [8-10]. These bands are conventionally called fluorescence of type $S_{2} \longrightarrow S_{0}$.
Since the nature of the excited states of PMD has not been studied, the reason for the fluorescence of dyes from higher excited states remains unclear. This paper presents a peculiar approach to solving this problem, which made it possible to explain the nature of the phenomenon. In this work, we performed the corresponding quantum-chemical studies that could solve this problem, namely, to obtain the physical picture of the effect, the number of the singlet excited state, its localization in the molecule and symmetry, the analysis of the probabilities of quantum transitions, and, as a result, allows explaining the effect.

\section{Objects and Methods of Research}

Consider two compounds whose fluorescence $S_{2} \longrightarrow S_{0}$ is described in the literature [8-10].

Returning to these works, we can find a modern explanation for abnormal fluorescence. First, consider the dye trimetin-di-(4-dimethylaminophenyl) (PMD-I) [8].

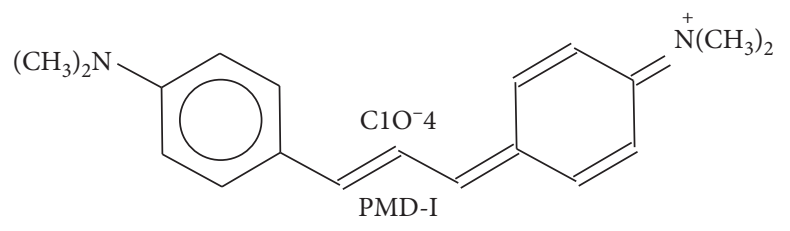

The next dye, which was studied in this paper, was PMD-II.

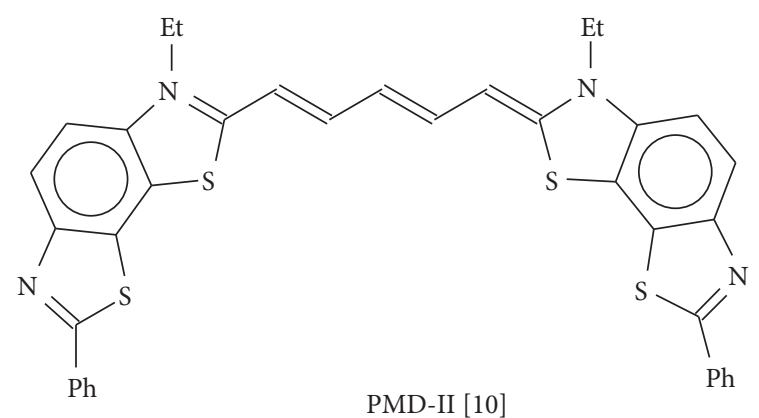

The absorption spectrum of PMD-I has the intense long wave band with $\lambda_{\max }=665 \mathrm{~nm}$ with vibrational repetition at $640 \mathrm{~nm}$ and a number of weak bands with maxima in the region of 440, 400, and $300 \mathrm{~nm}$ (Figure 1) [8, 11].

The research of the fluorescence of PMD-I showed that, in addition to the fluorescence band in the $700 \mathrm{~nm}$ region, there is a short-wave fluorescence band with a maximum at $490 \mathrm{~nm}$ (Figure 1). The research of the excitation spectrum of this fluorescence showed that it corresponds to the absorption spectrum in the region $\lambda_{\max }<450 \mathrm{~nm}$. The authors of [8], like other authors who studied this fluorescence, believe that an important feature of the dye absorption spectrum is the practical absence of the contribution from the electron-vibrational $S_{0} \longrightarrow S_{1}(v)$-transition to absorption in the region of the $S_{0} \longrightarrow S_{2}$-transition. This causes a significant decrease in the internal conversion of $S_{2} \longrightarrow S_{1}$ and the appearance of $S_{2} \longrightarrow S_{0}$-fluorescence. 


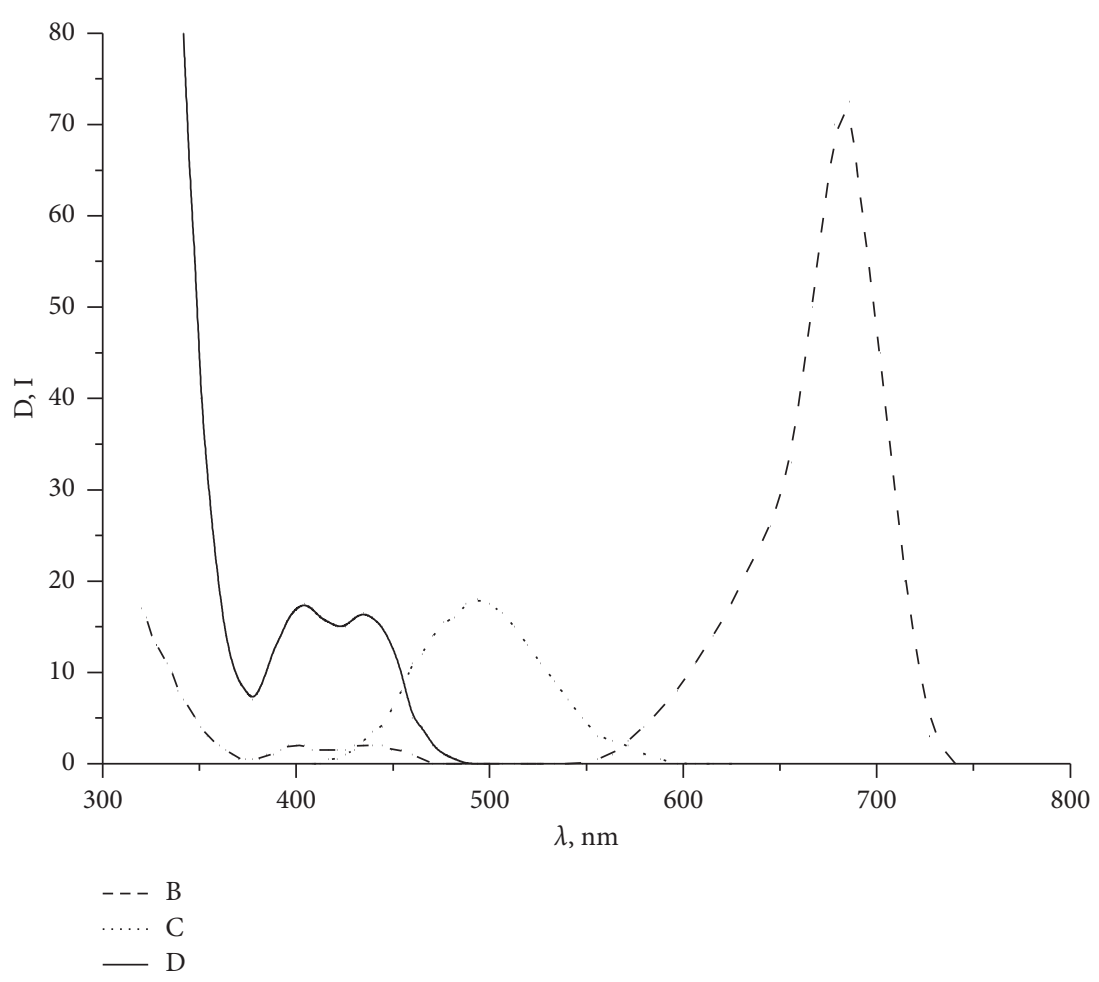

FIGURE 1: Absorption spectrum of trimethine-di-(4-dimethylaminophenyl) (B), the same spectrum with 10 times increased optical density $(\mathrm{D})$, and the spectrum of $(\mathrm{S})_{2} \longrightarrow(\mathrm{S})_{0}$-fluorescence $(\mathrm{C})$ [8].

Analogous results were obtained for the dye PMD-II [10], and it turned out that the intensity of $S_{2} \longrightarrow S_{0}$-fluorescence in this case is much higher than the azulene fluorescence intensity in the analogous quantum transition. However, the author of [10] paid attention to the fact that the absence of the contribution of the $S_{0} \longrightarrow S_{1}(\mathrm{v})$-transition into the absorption of the molecule in the region $S_{0} \longrightarrow S_{2}$ is not a sufficient condition for the appearance of $S_{2} \longrightarrow S_{0^{-}}$ fluorescence. This can be explained by the absence of shortwave fluorescence in cyanines, in which the energy distance between $S_{2}$ and $S_{1}$ is larger than that in the case of PMD-II.

Since the nature of the excited states of PMD has not been investigated, the cause of the fluorescence of the dye from higher excited states remains unclarified. Therefore, the corresponding quantum-chemical studies were carried out in this work in order to answer to the posed question.

In the electrically neutral state, the PMD-I and PMD-II dye molecules contain an odd number of electrons, that is, they are radicals, unstable formation. Stabilization of the molecule is achieved by transferring to the cation state. The anion, as a rule, is $\mathrm{ClO}_{4}^{-}$. This anion in the dye solutions does not create neutral associates. This would significantly change the physical properties of the dye.

For the research, quantum-chemical calculations using the HyperChem software package were used. In particular, semiempirical methods of calculating $\mathrm{MNDO}, \mathrm{MNDO} / \mathrm{d}$, and AM1 were used. These methods give a certain shift in the position of the bands in the absorption spectrum. Nevertheless, they give the correct order in the spectral arrangement of various quantum transitions and provide correct data on the oscillator strength in various quantum transitions and the energy difference between quantum transitions. Since the method AM1 proved to be convenient in use, the basic quantum-chemical studies were carried out with its use.

During the research, the authors found out that the effects of symmetry play a key role in the appearance of $S_{2} \longrightarrow S_{0}$-fluorescence. It turned out that the calculation procedure can give deviations of bond lengths in the third sign after the comma, which immediately reduces the symmetry of the molecule and distorts the form of the molecule orbitals (MOs). Therefore, special attention was paid to symmetrization of the geometric structure of PMD molecules.

To obtain a full answer to this question, group-theoretic analysis was used to conduct studies on the energy structure of molecules.

\section{The Results of the Study and Discussion}

3.1. Group-Theoretical Analysis. In this work, the energy structure and nature of the energy states of the PMD-I and PMD-II molecules were studied. To determine the true cause of the appearance of $S_{2} \longrightarrow S_{0}$-fluorescence, it is necessary to include studies of the symmetry of the energy states of excited molecules because the laws of symmetry are the most important ones in nature. Therefore, they are able to answer the posed question.

PMD-I and PMD-II cations in the free state were used in the study. The authors, in accordance with experimental data, believed that the presence of solvent molecules in the near environment to the dye molecule will slightly change the spectral properties of the dissolved dye molecule. 
The PMD-I and PMD-II cations in the trans-configuration are described by the symmetry group $C_{2 \mathrm{v}}$. Table 1 shows the results of the PMD-I analysis.

As follows from Table 1, the total number of degrees of freedom $(N=132)$ was distributed according to the representations of the group $C_{2 \mathrm{v}}: A_{1}-44, A_{2}-21, B_{1}-44$, and $B_{2}-23$. This information is important to know before studying the infrared absorption spectra of the PMD-I molecules. On the other hand, Table 1 presents data on the number and symmetry of molecular orbitals (MOs) of $\sigma$ and $\pi$-type. As seen from Table 1 , in the $C_{2 \mathrm{v}}$ group, the $\sigma$-MO and $\pi$-MO refer to different representations.

A similar description of the PMD-II molecule showed that, in this case, the total number of the degrees of freedom $(N=234)$ was distributed according to the representations: $A_{1}-78, A_{2}-38, B_{1}-76$, and $B_{2}-38$. These degrees of freedom include 3 translations, 3 rotations, 77 completely symmetric vibrations, 37 oscillations of symmetry $A_{2}, 76$ vibrations of symmetry $B_{1}$, and 38 vibrations of symmetry $B_{2}$.

On the other hand, chemical bonds between atoms form $78 \sigma$-MO and $41 \pi$-MO. There are 108 electrons on the MO occupied by electrons (electrons are placed on $1 s$ atomic orbitals of hydrogen atoms, on $2 s$ and $2 p$ atomic orbitals of $C$ and $N$ atoms, and also on $3 s$ and $3 p$ atomic orbitals of $S$ atoms). It is important that the $\sigma$-MO refers to the representations $A_{1}$ and $B_{1}$, while $\pi$-MO refers to the representations $A_{2}$ and $B_{2}$ of the symmetry group $C_{2 \mathrm{v}}$.

It is known from quantum mechanics that the probability of quantum transition of an electron between two MOs is described by means of the dipole moment of a quantum transition:

$$
D_{m n}^{\xi}=-e \int \psi_{m}^{*} \xi \psi_{n} \mathrm{~d} v
$$

where $\xi=x, y, z$ denotesmatrix element of the dipole electric moment vector; $\psi_{n}$ and $\psi_{m}$ are the $\psi$-functions of the electron in states with numbers $m$ and $n$.

To determine if the corresponding quantum transition is allowed, it is sufficient to consider this integral from the group theory point of view. In this case, wave functions and coordinates are substituted by their representations in the theory of symmetry. Afterwards, it is found out whether the corresponding product contains a fully symmetric representation. If there is no such content, the probability of a quantum transition is zero. Therefore, knowing the MO representation in the $C_{2 v}$ group, we can immediately find the presence of a quantum transition and its polarization. The corresponding information is presented in Table 2.

As follows from Table 2, the quantum transitions between two $\pi$-MOs are always allowed and polarized in the plane of the molecule along the $x$ or $y$ axis. Situation is similar between two $\sigma$-MOs. Also, quantum transitions $\pi \longleftrightarrow \sigma$ can be already polarized along the $z$ axis, or they are forbidden by symmetry.

Certainly, there can be a spatial prohibition in addition to the prohibition of quantum transitions by symmetry. It shows up in the case when MOs, between which the quantum transition of an electron is calculated, are spatially separated and do not overlap.
TABLE 1: Characters of the $C_{2 \mathrm{v}}$ group and assigning the coordinate axes, translations $(T)$, rotations $(R)$, the number of vibrational modes $(n)$, and $\sigma$-MO and $\pi$-MO of the PMD-I molecule to them.

\begin{tabular}{llllllllllll}
\hline$C_{2 \mathrm{v}}$ & $E$ & $C_{2}$ & $\sigma_{\mathrm{h}}$ & $\sigma_{\mathrm{v}}$ & & $T$ & $R$ & $N$ & $n$ & $\sigma$ & $\pi$ \\
\hline$A_{1}$ & 1 & 1 & 1 & 1 & $y$ & 1 & 0 & 44 & 43 & 23 & 0 \\
$A_{2}$ & 1 & 1 & -1 & -1 & & 0 & 1 & 21 & 20 & 0 & 8 \\
$B_{1}$ & 1 & -1 & 1 & -1 & $x$ & 1 & 1 & 44 & 42 & 22 & 0 \\
$B_{2}$ & 1 & -1 & -1 & 1 & $z$ & 1 & 1 & 23 & 21 & 0 & 9 \\
$X$ & 1 & -1 & 1 & -1 & & & & & & & \\
$Y$ & 1 & 1 & 1 & 1 & & & & & & & \\
$Z$ & 1 & -1 & -1 & 1 & & & & & & & \\
$T$ & 3 & -1 & 1 & 1 & & & & & & & \\
$R$ & 3 & -1 & -1 & -1 & & & & & & & \\
$N$ & 132 & -2 & 44 & 2 & & & & & & & \\
$\sigma-\mathrm{MO}$ & 44 & 2 & 44 & 2 & & & & & & & \\
$\pi-\mathrm{MO}$ & 17 & -1 & -17 & 1 & & & & & & & \\
\hline
\end{tabular}

TABLE 2: Quantum transitions allowed by the symmetry group $C_{2 \mathrm{v}}$ with absorption or emission of light.

\begin{tabular}{lllll}
\hline $\mathrm{MO}$ & $\sigma-\mathrm{A}_{1}$ & $\pi-\mathrm{A}_{2}$ & $\sigma-\mathrm{B}_{1}$ & $\pi-\mathrm{B}_{2}$ \\
\hline$\sigma-\mathrm{A}_{1}$ & $y-A_{1}$ & $-\mathrm{A}_{2}$ & $x-\mathrm{B}_{1}$ & $z-\mathrm{B}_{2}$ \\
$\pi-\mathrm{A}_{2}$ & $-\mathrm{A}_{2}$ & $y-A_{1}$ & $z-\mathrm{B}_{2}$ & $x-\mathrm{B}_{1}$ \\
$\sigma-\mathrm{B}_{1}$ & $x-\mathrm{B}_{1}$ & $z-\mathrm{B}_{2}$ & $y-A_{1}$ & $-\mathrm{A}_{2}$ \\
$\pi-\mathrm{B}_{2}$ & $z-\mathrm{B}_{2}$ & $x-\mathrm{B}_{1}$ & $-\mathrm{A}_{2}$ & $y-A_{1}$ \\
\hline
\end{tabular}

It is important to pay attention to one more circumstance in the future. There are nonradiative transitions besides radiative ones. In this case, the electron excitation energy is converted into the thermal energy of the solution. The probability of such transition (internal conversion) can be found if the operator of the perturbation which causes a conversion is known:

$$
p=\left|\int \psi_{S_{1}} p \psi_{S_{0}} \mathrm{~d} v\right|^{2}
$$

Assuming the operator responsible for the nonradiative transition is symmetric, we find the selection rules for such transitions: in the $c_{2 v}$ group, nonradiative transitions between MOs of the same symmetry are allowed. In addition, it turned out that internal conversion is inefficient for those transitions that do not change the interatomic distance in the molecule. Also, since the interatomic distance changes markedly under excitations into the $\sigma^{*}$-state $\left(\pi \longrightarrow \sigma^{*}\right.$ and $\left.\sigma \longrightarrow \sigma^{*}\right)$, in these cases, the internal conversion efficiency increases significantly [11].

3.2. Molecule PMD-I. Calculation of the energy structure of PMD-I was conducted taking into account the configuration interaction between 12 occupied MOs and 12 free MOs (CI $12 \times 12$ ). The calculated absorption spectrum is shown in Figure 2 .

The results of the calculation of the energy structure, as well as the structure and symmetry of the MO, are given in Table 3.

Table 3 contains data only for those singlet-triplet transitions in which energy is lower than the singlet-singlet transition energy. Two quantum transitions are indicated for 


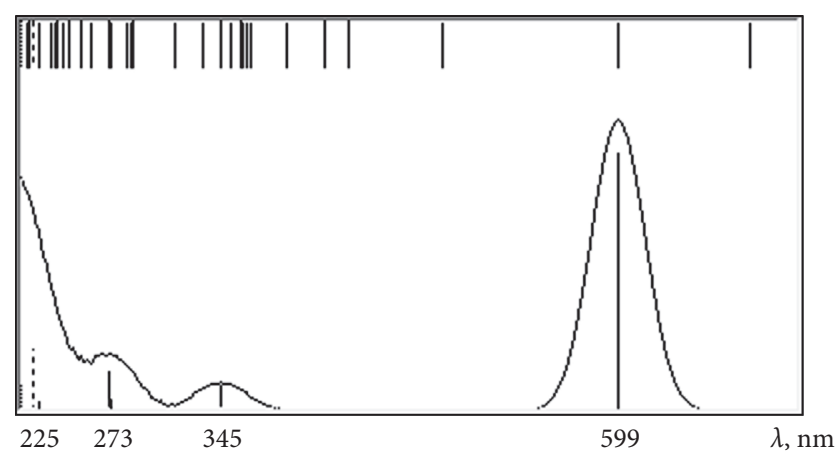

Figure 2: The absorption spectrum of PMD-I. It is calculated using the AM1 method considering the CI $12 \times 12$. All singlet and triplet quantum transitions (except the first one) are noted at the top without taking into account the oscillator strength.

some absorption bands. The configurational interaction between these transitions ensures the formation of a corresponding absorption band.

The comparison of the data depicted in Figure 2 and in Table 3 reveals that the quantum transitions $S_{0} \longrightarrow S_{2}$ and $S_{0} \longrightarrow S_{3}$ are weak and imperceptible against the background of the other absorption bands. The oscillator strength in these bands may change somewhat under the influence of the solvent, but they will still remain weak.

We consider the form of the corresponding molecular orbitals to understand the reason why the oscillator strength in the second and third absorption bands is small.

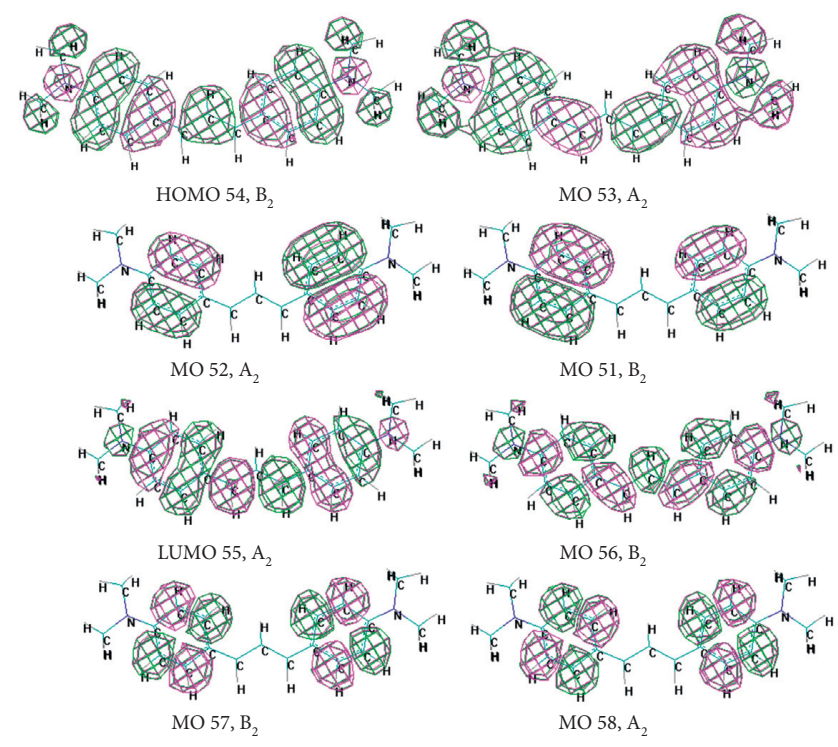

The quantum transition $S_{0} \longrightarrow S_{2}$ is allowed by symmetry. However, the structure of the MO 51 and 58, which provides this transition, leads to strictly identical positive and negative components in the integrand for the dipole moment of the transition. This leads to the fact that the oscillator strength becomes less than $10^{-4}$. The reason for the small oscillator strength of the $S_{0} \longrightarrow S_{3}$ transition is the same. In this case, the positive and negative components of the integrand do not compensate each other completely. This is the main reason for the absence of the energy exchange between the $S_{3^{-}}$and $S_{2}$-energy states and the $S_{3}$ - and $S_{1}$-energy states. In the latter case, the internal conversion is forbidden, while the excitation relaxation by the dipole mechanism (the radiative process) is very weak.

3.3. The PMD-II Molecule. The geometric structure of the PMD-II molecule is much more complicated than PMD-I. However, the calculated absorption spectra of PMD-II (Figure 3) and PMD-I qualitatively resemble each other.

Details of the calculations of the energy structure for the molecule PMD-II, as well as the structure and symmetry of the MO, are given in Table 4.

It follows from Table 4 that there are two more quantum transitions with the formation of singlet excited states between the $S_{0} \longrightarrow S_{1}$ and $S_{0} \longrightarrow S_{4}$ transitions, which are visible in the absorption spectrum (Figure 3 ). However, both transitions correspond to the excitation of an electron from the $\pi$-system to the $\sigma$-system. In this case, it is clear that the first of them $\left(S_{0} \longrightarrow S_{2}\right)$ is forbidden in symmetry and the second one $\left(S_{0} \longrightarrow S_{3}\right)$ is forbidden in space.

Considering the effect of symmetry on the relaxation processes in the PMD-II molecule, it can be concluded that both the abovementioned excitation relaxation mechanisms are forbidden for the transition from $\mathrm{S}_{4}$-state to the $S_{2}$-state. The excitation relaxation from $S_{4}$ to $S_{3}$ is allowed by the first mechanism although it is slowed down due to the spatial prohibition. Energy exchange between $S_{3}$ and $S_{2}$ states is possible, but it does not affect the course of further excitation relaxation processes. The excitation relaxation from $S_{3}$ to $S_{1}$ is forbidden by both the mechanisms. The radiative process $S_{3} \longrightarrow S_{0}$ is allowed by symmetry.

Another interesting parallel is observed for the two molecules considered. There are two closely placed energy states between the states $S_{1}$ and $S_{4}$, the transitions to which are characterized by small values of the oscillator strength. In PMD-I, this is $\pi \pi^{*}$-state, while in PMD-II, this is $\pi \sigma^{*}$-state. The reason for this similarity is the structure of the MO, to which the electron is transferred from the ground state. In both cases, the MOs are divided into two remote parts. If one $\mathrm{MO}$ is represented as the sum of the right and left parts, then 
TABLe 3: Calculated absorption spectrum of PMD-I.

\begin{tabular}{|c|c|c|c|c|}
\hline Type of quantum transition & Wavelength $\lambda, \mathrm{nm}$ & MO, between which there was a transition & Oscillator strength & Symmetry \\
\hline$S_{0} \longrightarrow T_{1}$ & 1146.8 & $54 \longrightarrow 55$ & 0 & $B_{2} \longrightarrow A_{2}-x-B_{1}$ \\
\hline$S_{0} \longrightarrow T_{2}$ & 684.3 & $\begin{array}{l}54 \longrightarrow 56 \\
53 \longrightarrow 55\end{array}$ & 0 & $\begin{array}{l}\mathrm{B}_{2} \longrightarrow \mathrm{B}_{2}-y-A_{1} \\
\mathrm{~A}_{2} \longrightarrow \mathrm{A}_{2}-y-A_{1}\end{array}$ \\
\hline$S_{0} \longrightarrow S_{1}$ & 598.9 & $54 \longrightarrow 55$ & $\begin{array}{l}1.5975 \\
\pi \longrightarrow \pi\end{array}$ & $B_{2} \longrightarrow A_{2}-x-B_{1}$ \\
\hline$S_{0} \longrightarrow S_{2}$ & 351.7 & $\begin{array}{l}54 \longrightarrow 58 \\
51 \longrightarrow 55\end{array}$ & $\begin{array}{l}0.0000 \\
\pi \longrightarrow \pi\end{array}$ & $\begin{array}{l}B_{2} \longrightarrow A_{2}-x-B_{1} \\
B_{2} \longrightarrow A_{2}-x-B_{1}\end{array}$ \\
\hline$S_{0} \longrightarrow S_{3}$ & 351.5 & $\begin{array}{l}54 \longrightarrow 57 \\
52 \longrightarrow 55\end{array}$ & $\begin{array}{l}0.0011 \\
\pi \longrightarrow \pi\end{array}$ & $\begin{array}{l}\mathrm{B}_{2} \longrightarrow \mathrm{B}_{2}-y-A_{1} \\
\mathrm{~A}_{2} \longrightarrow \mathrm{A}_{2}-y-A_{1}\end{array}$ \\
\hline$S_{0} \longrightarrow S_{4}$ & 344.9 & $\begin{array}{l}54 \longrightarrow 56 \\
53 \longrightarrow 55\end{array}$ & $\begin{array}{l}0.1673 \\
\pi \longrightarrow \pi\end{array}$ & $\begin{array}{l}\mathrm{B}_{2} \longrightarrow \mathrm{B}_{2}-y-A_{1} \\
\mathrm{~A}_{2} \longrightarrow \mathrm{A}_{2}-y-A_{1}\end{array}$ \\
\hline
\end{tabular}

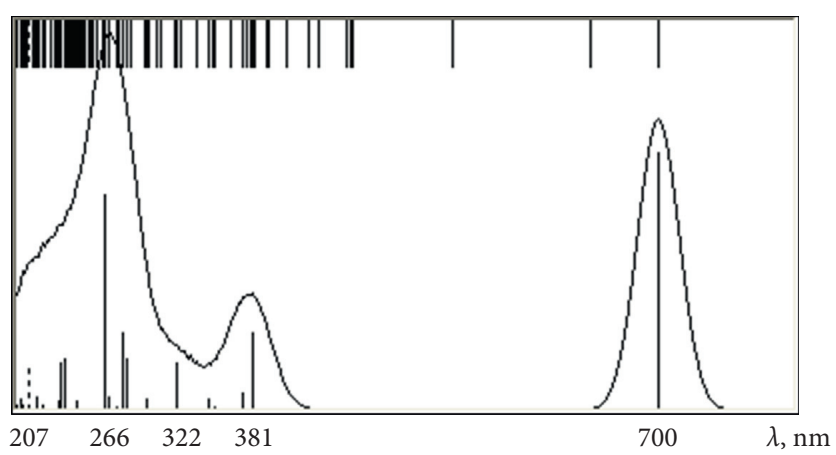

FIgURE 3: The calculated absorption spectrum of PMD-II.

TABLE 4: Calculated absorption spectrum of PMD-II.

\begin{tabular}{|c|c|c|c|c|}
\hline Type of quantum transition & Wavelength $\lambda, \mathrm{nm}$ & MO, between which transition occurred & Oscillator strength & Symmetry \\
\hline$S_{0} \longrightarrow T_{1}$ & 1389.3 & $110 \longrightarrow 111$ & 0 & $B_{2} \longrightarrow A_{2}-x-\mathrm{B}_{1}$ \\
\hline$S_{0} \longrightarrow T_{2}$ & 647.6 & $\begin{array}{l}110 \longrightarrow 112 \\
107 \longrightarrow 111\end{array}$ & 0 & $\begin{array}{l}B_{2} \longrightarrow B_{2}-y-A_{1} \\
A_{2} \longrightarrow A_{2}-y-A_{1}\end{array}$ \\
\hline$S_{0} \longrightarrow S_{1}$ & 700.2 & $110 \longrightarrow 111$ & $\begin{array}{l}1.5603 \\
\pi \longrightarrow \pi\end{array}$ & $B_{2} \longrightarrow A_{2}-x-B_{1}$ \\
\hline$S_{0} \longrightarrow S_{2}$ & 456.2 & $110 \longrightarrow 116$ & $\begin{array}{c}0.0002 \\
\pi \longrightarrow \sigma\end{array}$ & $B_{2} \longrightarrow \mathrm{B}_{1}-\mathrm{A}_{2}$ \\
\hline$S_{0} \longrightarrow S_{3}$ & 456.0 & $110 \longrightarrow 115$ & $\begin{array}{l}0.0042 \\
\pi \longrightarrow \sigma\end{array}$ & $B_{2} \longrightarrow \mathrm{A}_{1}-z-\mathrm{B}_{2}$ \\
\hline$S_{0} \longrightarrow S_{4}$ & 381.4 & $\begin{array}{l}110 \longrightarrow 112 \\
107 \longrightarrow 111\end{array}$ & $\begin{array}{c}0.4731 \\
\pi \longrightarrow \pi\end{array}$ & $\begin{array}{l}B_{2} \longrightarrow B_{2}-y-A_{1} \\
A_{2} \longrightarrow A_{2}-y-A_{1}\end{array}$ \\
\hline
\end{tabular}

the second one will correspond to the difference of the same parts. The distance between them causes a very small splitting between $S_{2^{-}}$and $S_{3}$-states.

Consequently, the quantum transition $S_{3} \longrightarrow S_{0}$ is responsible for the PMD fluorescence. Experimental studies have shown that the quantum yield of $S_{3} \longrightarrow S_{0}$-fluorescence rarely exceeds $1 \%$. Thus, even with a weak overlap of the spectra of $S_{0} \longrightarrow S_{1}(v)$ - and $S_{0} \longrightarrow S_{3}$-absorbances, the internal conversion $\left(S_{3} \longrightarrow S_{1}\right)$ proceeds at a rate that is two orders of magnitude greater than the speed of the radiative process $S_{3} \longrightarrow S_{0}$.

\section{Conclusions}

Based on the conducted studies aimed at elucidating the mechanisms of fluorescence of PMD from higher excited states, the following conclusions are made:

(1) Investigation of PMD from different groups of dyes with the molecules giving fluorescence from higher excited states was conducted. It was showed that, for both cases, there are two more excited states with low oscillator strengths for quantum transitions $S_{0} \longrightarrow S_{2}$ 
and $S_{0} \longrightarrow S_{3}$ in the transparency region of the PMD solution between the first and fourth excited states. In this case, the oscillator strength of the $S_{0} \longrightarrow S_{3}$ transition is more than an order of magnitude greater than the oscillator strength of the $S_{0} \longrightarrow S_{2}$ transition.

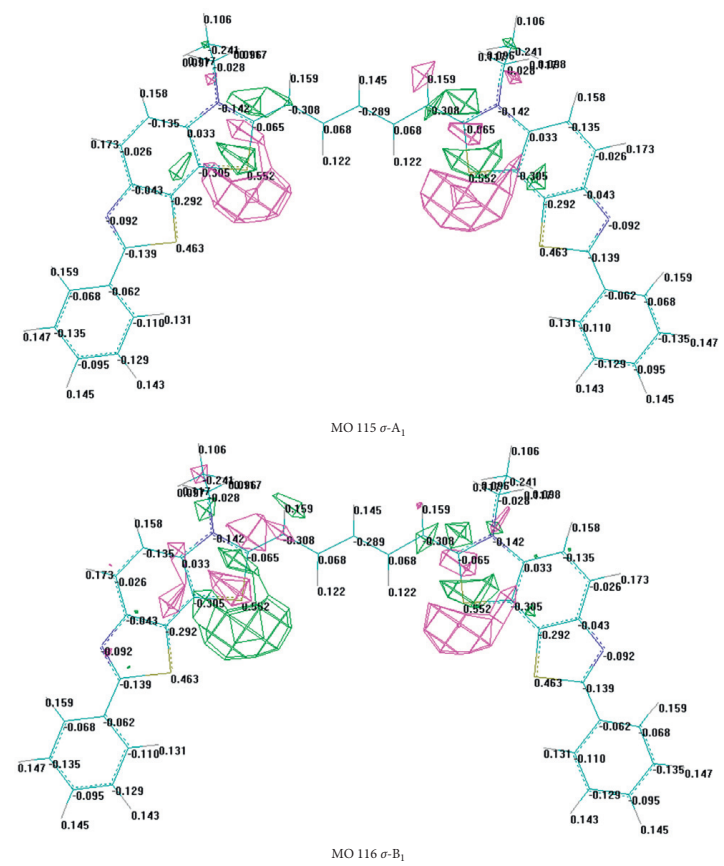

(2) In the case of PMD-I, the transitions $S_{0} \longrightarrow S_{2}$ and $S_{0} \longrightarrow S_{3}$ correspond to the excitation of an electron within the $\pi$-electron system. In the case of PMD-II, excitations into $S_{2^{-}}$and $S_{3}$-states correspond to $\pi \longrightarrow \sigma^{*}$-quantum transitions. The common thing for both dyes is the structure of the MO of excited states, which consists of two isolated regions of localization of the electron. One of the MO of excited states can be represented as the sum of two parts, and the second as a difference between them.

(3) The group-theoretical analysis of PMD showed that relaxation processes between the states $S_{3}$ and $S_{1}$ are forbidden, either by radiation or by internal conversion. Only transition to the ground state from the state $S_{3}$ of the molecule is possible. Since the experimental data state that the quantum yield of $S_{3} \longrightarrow S_{0}$ fluorescence does not exceed $1 \%$, it is indicated that the internal conversion rate can be 2 orders of magnitude higher than the radiative relaxation rate of the molecule.

(4) It can be asserted that the necessary condition for the appearance of fluorescence from the higher excited states of molecules $S_{3} \longrightarrow S_{0}$ is the absence of $S_{0} \longrightarrow S_{1}(v)$-absorption in the region of the $S_{0} \longrightarrow S_{3}$ transition. Whereas, the sufficient condition is the corresponding symmetry of the excited states, which imposes a prohibition on the $S_{3} \longrightarrow S_{1}$ relaxation process.

\section{Data Availability}

The data used to support the findings of this study are available from the corresponding author upon request.

\section{Additional Points}

The HyperChem Molecular Modeling System software package was used in this work (http://www.hyper.com/? tabid=360). Quantum chemical calculations were performed by the methods described in https://www.scribd. com/document/380681457/HyperChem-Professional-8-0Manual-de-Utilizare\#download.

\section{Conflicts of Interest}

The authors declare that they have no conflicts of interest.

\section{Acknowledgments}

The authors are thankful to Rector SumSU and Rector NAU for their interest and permission for experimental and publication purposes.

\section{References}

[1] G. Viswanath and M. Kasha, "Confirmation of the anomalous fluorescence of azulene," The Journal of Chemical Physics, vol. 24, no. 3, pp. 574-577, 1956.

[2] R. König, D. Leupold, B. Voigt, and S. Dähne, "Anomalous fluorescence behaviour of organic dyes," Journal of Luminescence, vol. 9, no. 2, pp. 113-120, 1974.

[3] M. Sakamoto, X. Cai, M. Hara, S. Tojo, M. Fujitsuka, and T. Majima, "Anomalous fluorescence from the azaxanthone ketyl radical in the excited state," Journal of the American Chemical Society, vol. 127, no. 11, pp. 3702-3703, 2005.

[4] J. S. de Klerk, A. Szemik-Hojniak, F. Ariese, and C. Gooijer, "Intramolecular proton-transfer processes starting at higher excited states: a fluorescence study on 2-Butylamino-6methyl-4-nitropyridineN-Oxide in nonpolar solutions," The Journal of Physical Chemistry A, vol. 111, no. 26, pp. 58285832, 2007.

[5] V. P. Kazakov, S. S. Ostakhov, and I. O. Osina, "Violation of Vavilov's law for the "Anomalous" S $2 \longrightarrow$ S 0 fluorescence of tryptophan in complexes with europium(III) in aqueous ethanolic solutions," High Energy Chemistry, vol. 43, no. 1, pp. 64-66, 2009.

[6] C. L. Choi, H. Li, A. C. K. Olson, P. K. Jain, S. Sivasankar, and A. P. Alivisatos, "Spatially indirect emission in a luminescent nanocrystal molecule," Nano Letters, vol. 11, no. 6, pp. 2358-2362, 2011.

[7] T. Itoh, "Fluorescence and phosphorescence from higher excited states of organic molecules," Chemical Reviews, vol. 112, no. 8, pp. 4541-4568, 2012.

[8] E. A. Tikhonov, O. V. Przhonskaya, and M. T. Shpak, "Pogloshcheniye I fluorestsentsiya pri perehodah iz vysokovozbuzhdyonnyh elektronnyh sostoyaniy polimetinovyh krasiteley," Kvantovaya Elektronika, vol. 10, pp. 92-108, 1987, in Russian.

[9] G. G. Dyadyusha, O. V. Przhonskaya, E. A. Tihonov et al., "Intense fluorescence from the second excited state of molecular solutions of organic dyes," JETP Letters (Letters to 
Journal of Experimental and Theoretical Physics), vol. 14, no. 5, pp. 220-222, 1971.

[10] A. A. Ishchenko, "Stroenie i spektral'no-lyuminestsentnye svoystva polimetinovyh krasiteley," Uspekhi Khimii, vol. 60, no. 8, pp. 1708-1743, 1991.

[11] P. O. Kondratenko, Fotohimichna Diya Svitla, University inKiev, Kiev, Ukraine, 2005, in Ukrainian. 